\title{
The Creatures of Flame: Richard Wrangham's Catching Fire
}

\author{
Catching Fire: How Cooking Made Us Human, by Richard Wrangham. Philadelphia: \\ Perseus, 2009. Pp. 309. \$26.95
}

Haley Moss Dillon • Rachael A. Carmen • Glenn Geher

Published online: 18 January 2011

(C) Springer Science+Business Media, LLC 2011

Where do we come from? At this point in human history, the best evidence suggests that evolution by natural selection has been the dominant force in shaping human's cognitive processes and physical structures (Buss 2004). In making the case that processes connected to cooking hold the true key to human origins, Richard Wrangham (2009) begins his fascinating account deep in time in the jungles of Africa. From Wrangham's perspective, evolution has touched all aspects of what it means to be human. In particular, Wrangham evaluates a daily process, cooking, in terms of its evolutionary service. From bipedalism to our large brains, cooking has affected the very essence of what separates humans from other species. Wrangham's major hypothesis is coined "the cooking hypothesis," and is centered around our adapted diet of cooked food, and how the results of cooking pervade our lives-from our bodies to our minds (p. 14). Wrangham argues that the advent of fire, and cooking (as a result), gave rise to the genus Homo (p. 2).

\section{The Cook's Body}

Limited ability to process raw food stems from our relatively small digestive system, but this same small digestive system enables us to process cooked food with

H. M. Dillon $(\bowtie) \cdot$ R. A. Carmen · G. Geher

SUNY New Paltz,

314 JFT, 1 Hawk Dr.,

New Paltz, NY 12561, USA

e-mail: Haleymoss@gmail.com exceptional proficiency (p. 38). Cooked food additionally can promote more efficient growth, as demonstrated by domesticated animals (generally on farms) that grow faster and "better" on cooked food diets. Wrangham states "whether domestic or wild, mammal or insect, useful or pest, animals adapted to raw diets tend to fare better on cooked food" (pp. 39-40).

Wrangham proposes a theory as to why humans are "brainier" than other species, which is rooted in evolutionary logic. Darwin concluded that intellect would have certain advantages in social competition, and the major reason for a species difference in intellect is how much brainpower the body is able to afford. For this reason, Wrangham postulates diet as a key driver for the growth of primate brains - for humans, cooking must have played a major role (p. 105). Creationists are known for the phrase "man was created in God's own image"-a creationist argument has been that if we were not created by God in his image, then why are humans so advanced in comparison to the rest of the animal kingdom? Wrangham's major argument throughout the work is that fire, or, rather, the application of fire to food is what separates us from the rest of the animal kingdom.

Evolutionary benefits stem from the fact that digestion is a very costly process, which takes up a large proportion of an individual's energy budget, often as much as moving does. Once cooked food became an everyday thing, natural selection favored those with smaller guts, due to their ability to digest food well, but faster and at a lower cost than before. How is this adaptive? Lower cost digestion results in increased energetic efficiency (p. 40). Energetic efficiency refers to the biological components of digestion: foods that are digested between our mouths and our small intestines produce calories, which are available for our use, whereas the foods that are digested by our intestinal flora 
yield only a fraction of their available energy to us (p. 58). Thus, if food is easier to digest (a fact of cooked versus raw food), we gain use of calories that would otherwise have been absorbed during the digestion process.

An interesting way to conceptualize cooking as an adaptation is to see cooking as technologically externalizing part of our digestion process (p. 56). Wrangham believes the effects of cooking on energy are consistently positive, despite claims of some nutritionists and raw foodists. Wrangham goes on to describe the biological processes of digestion, giving the reader a scientific basis by which to compare his theories.

What brings Wrangham's work to the next level is its interdisciplinary scope - a feature common among the work of evolutionists-as evolutionary scholarship naturally bridges disciplines (see Wilson et al. 2009). In his account of how cooking has come to shape the nature of humans, he makes the case that cooking has shaped humanity writ large - affecting not only our bodies, but our minds and social structures as well. In making this case, Wrangham draws on literature broadly - including cultural anthropology, biological anthropology, evolutionary psychology, comparative physiology, and more. Clearly, Wrangham naturally connects multiple disciplines in his scholarship - an outcome that owes largely to his keen intellect-but that also owes to the powerfully integrative nature of evolutionary theory as academic tool sine qua non (see Garcia et al. 2011).

\section{Evolution in Action-Continuing to Explain Who We Are}

Wrangham has contributed another piece of the evergrowing puzzle that explains humanity. By discussing the elements of cooked food (which have led to the modern humans), Wrangham is providing evolutionists with answers to questions plaguing the modern scientist. Further, cooking food is somewhat of a cultural innovation, suggesting that our cultural means have pushed us across the species threshold, into a modern society. Typically, culture has been fundamentally separate from science and nature, but Wrangham's theory on the creatures of flame bridges that separation.

There are many evolutionary and educational messages in this book that are relevant to everyday life. For instance, the cooking hypothesis could be integrated into an evolutionary fitness class used as part of an evolutionary studies program. By coupling the advent of fire and the evolution of Homo sapiens 200,000+ years ago, we can better understand modern day humans - from our digestive system to our large brains. Never before has someone solidified an evolutionary theory (based on diet alone) regarding what truly separates Homo sapiens from the rest of the animal kingdom. We know that humans possess unique attributes that separate us from other animals (i.e., language) - and after reading Dr. Wrangham's book, we are convinced that cooking should be included on that list. Granted, Wrangham's cooking hypothesis is still in its infancy, but with a little more supplemental research, his hypothesis could be one of the major points that is touched upon when discussing the evolutionary divergence between modern day Homo sapiens and the ancestral genus we originated from. Food is such an important part of our everyday lives, but we never stop to ask ourselves how much the advent of cooked food helped shape our everyday lives. It is really up to the reader as an individual to decide whether they agree with Wrangham's cooking hypothesis, but personally, we will never look at cooked food the same way.

\section{References}

Buss DM (2004) Evolutionary psychology. In: Gregory RL, editor. Oxford companion to the mind, 2nd edition, Oxford.

Garcia J, Geher G, Crosier B, Saad G, Gambacorta D, Johnsen L, et al. (2011) The interdisciplinarity of evolutionary approaches to human behavior: a key to survival in the ivory archipelago. Futures (in press)

Wilson DS, Geher G, Waldo J. EvoS: completing the evolutionary synthesis in higher education. EvoS Journal: The Journal of the Evolutionary Studies Consortium. 2009;1:3-10.

Wrangham R. Catching fire: how cooking made us human. Philadelphia, PA: Perseus Books Group; 2009. 